\title{
Comparing complications of small-bore chest tubes to large-bore chest tubes in the setting of delayed hemothorax: a retrospective multicenter cohort study
}

\author{
A. Orlando 1,2,3,4,5,6, J. Cordero², M. M Carrick ${ }^{3}$ A. H. Tanner $\|^{4}$, K. Banton' ${ }^{1}$, R. Vogel ${ }^{2}$, M. Lieser ${ }^{5}$, D. Acuna ${ }^{6}$ and \\ D. Bar-Or ${ }^{1,2,3,4,5,6^{*}}$
}

\begin{abstract}
Background: Previous studies have provided initial data suggesting that small-bore ( $\mathrm{SB}, \leq 14 \mathrm{Fr}$ ) chest tubes have the same efficacy as large-bore ( $L B,>14 \mathrm{Fr}$ ) chest tubes for acute hemothorax (HTX), but data continue to be lacking in the setting of delayed HTX. This study compared complications of SB chest tubes to LB tubes in patients with delayed HTX.

Methods: This was a retrospective observational study across $7.5 \mathrm{yrs}$. at 6 Level 1 trauma centers. Patients were included if 1) diagnosed with a HTX or $>1$ rib fracture with bloody effusion from chest tube; 2 ) initial chest tube placed $\geq 36 \mathrm{~h}$ of hospital admission. Patients were excluded for hemopneumothoraces. The primary endpoint was having at least one of the following chest tube complications: tube replacement, VATS, tube falling out, tube clogging, pneumonia, retained HTX, pleural empyema. Secondary outcomes included chest tube output volume and drainage rate. Dependent/independent and parametric/non-parametric analyses were used to assess primary and secondary outcomes.
\end{abstract}

Results: There were 160 SB patients (191 tubes) and 60 LB patients (72 tubes). Both comparison groups were similar in multiple demographic, injury, clinical features. The median (IQR) tube size for each group was as follows: SB [12 Fr (12-14)] and LB [32 Fr (28-32)]. The risk of having at least one chest tube complication was similar for LB and SB chest tubes (14\% vs. $18 \%, p=0.42)$. LB tubes had significantly larger risk of VATS, while SB tubes had significantly higher risk of pneumonia. SB tubes had significantly slower least squares (LS) mean initial output drainage rate compared to $L B$ tubes ( 52.2 vs. $213.4 \mathrm{~mL} /$ hour, $p<0.001$ ), but a non-parametric analysis suggested no significant difference in median drainage rates between groups 39.7 [23.5-242.0] mL/hr. vs. 38.6 [27.5-53.8], $p=0.81$. LB and SB groups had similar initial output volume (738.0 mL vs. 810.9, $p=0.59)$.

(Continued on next page)

\footnotetext{
* Correspondence: Davidbme49@gmail.com

'St. Anthony Hospital, Lakewood, CO, USA

${ }^{2}$ Swedish Medical Center, Englewood, CO, USA

Full list of author information is available at the end of the article
} 
(Continued from previous page)

Conclusions: There was no clearly superior chest tube diameter size; both chest tube sizes demonstrated risks and benefits. Clinicians must be aware of these potential tradeoffs when deciding on the diameter of chest tube for the treatment of delayed HTXs.

Keywords: Chest tube, Small-bore, Large-bore, Hemothorax, Complications

\section{Background}

With 300,000 cases annually and causing nearly $25 \%$ of deaths in the US trauma population, thoracic trauma is a frequent and serious condition $[1,2]$. Thoracic trauma can result in acute hemothoraces (HTXs), and due to their life-threatening nature, nearly all will require an emergent tube thoracostomy. On the other hand, delayed HTXs present later, occurring in $7-12 \%$ of thoracic trauma patients, with risks of chest tube thoracostomy ranging between 80 and 100\% [3, 4].

Delayed, or non-emergent HTXs are different from acute HTX in that they can be initially so small they do not warrant intervention, or they can be absent on initial chest computed tomography imaging. Although the standard of care for an acute traumatic HTX is the use of a large-bore (LB) 32-36 French (Fr) chest tube, [5] there is no clear chest tube size prescribed for the management of a delayed HTX. Because medicine has become increasingly palliative, some have questioned the practice of using LB chest tubes for the management of thoracic injuries, including HTXs and penumothoraces [6-15].

Anecdotally, the reluctance to utilize small-bore (SB) chest tubes for the treatment of delayed HTXs is due to the belief that, compared to LB chest tubes, SB tubes fall out more often and do not drain the HTX as quickly. On the other hand, it has been argued that SB chest tubes might be superior to LB tubes through decreased tube failure and patient pain [15-17]. Until recently, there has been little published on the topic, leaving clinicians with minimal evidence to substantiate the safety and efficacy of SB chest tubes for delayed HTXs.

Some studies have capitalized on this gulf of information and provided meaningful information. Niinami and colleagues demonstrated that despite the 28 Fr chest tube having a 9-fold greater draining capacity in the in vitro model compared to the $19 \mathrm{Fr}$ tube, the $19 \mathrm{Fr}$ tube drained equally well to the $28 \mathrm{Fr}$ tube in the in vitro porcine models [18]. In a 7-year study comparing $14 \mathrm{Fr}$ to $32-40 \mathrm{Fr}$ chest tubes for delayed HTXs and hemopneumothoraces, both the failure rates, median initial output, and insertion-related complications were all not significantly different between tube sizes [6]. A similar study compared 28-32 Fr versus 36-40 Fr chest tubes for HTXs and also reported no significant differences in initial output, duration of tube placement, or in the tube complication rates; moreover, the mean pain score was smaller for 28-32 Fr versus 36-40 Fr chest tubes [7]. Notwithstanding the growing literature, there continues to be a lack of studies examining small-bore chest tubes in the setting of delayed HTX. The objective of this study was to describe the safety and efficacy of SB $(\leq 14 \mathrm{Fr})$ chest tubes to LB ( $>14 \mathrm{Fr})$ chest tubes in the setting of delayed HTXs.

\section{Methods}

\section{Settings and patient population}

This was a retrospective observational cohort study utilizing data from six Level 1 Trauma Centers in the Injury Outcomes Network (www.ionresearch.org). Data were obtained via manual chart abstraction and trauma registries. Based on the 2012 Kulvatunyou et al. study, the current study was powered to detect an estimated difference of $16 \%$ between comparison groups in the primary outcome. Based on this sample size calculation, for a comparison of two proportions using Pearson's Chisquare statistic with a Chi-square approximation with a two-sided significance level of 0.05 , a sample size of 82 patients per group would achieve a power of at least 0.80 if the proportion of outcome events between groups is different by $16 \%$.

All patients included were admitted between 1/1/2010 and $06 / 30 / 2017$, had an age $\geq 18$ years, suffered a traumatic HTX (ICD-9: 860.2, 860.3; ICD-10: S27.1XXA) or had multiple rib fractures (ICD-9: 807.0-807.19; ICD-10: S22.31XA, S22.31XB, S22.32XA, S22.32XB, S22.39XA, S22.39XB, S22.41XA, S22.41XB, S22.42XA, S22.42XB, S22.43XA, S22.43XB, S22.49XA, S22.49XB) with bloody effusion (captured in medical records), and had an initial chest tube placed $\geq 36 \mathrm{~h}$ from hospital arrival. The time cutoff of $\geq 36 \mathrm{~h}$ was used to identify delayed HTXs. Chest tubes were excluded if placed for hemopneumothoraces or pneumothoraces. Patients with bilateral chest tubes with different categories of chest tube size on each side were excluded (e.g., SB on left side with LB on right side). This study was approved by each facility's institutional review board and was granted a waiver of informed consent and HIPAA authorization.

\section{Comparison groups}

The two comparison groups for this study were patients with delayed HTXs who were treated with SB chest 
tubes, and those treated with LB chest tubes. Small-bore chest tubes were defined as those with a diameter $\leq 14$ Fr, while LB chest tubes sizes were those with a diameter $>14 \mathrm{Fr}$ [8]. Defining SB chest tubes by the $\leq 14 \mathrm{Fr}$ cutoff is arbitrary, and was done to aid in comparing the results of the current study to existing studies $[6,8,10]$. All chest tubes were drained via gravity. Patients with SB chest tubes were primarily contributed by one facility that routinely placed them for the management of delayed HTXs. Patients with LB chest tubes were contributed by the remaining five centers that routinely placed them for delayed HTXs. Theoretically, the exchangeability assumption between the comparison groups in this study should hold because treatment with either SB or LB chest tubes was driven by a difference in geographic location, not due to demographic, clinical, or injury characteristics of each patient.

\section{Covariates}

The following demographic and injury characteristics were examined: sex (male, female), age, injury type (blunt, penetrating), highest chest abbreviated injury scale score (AIS), injury severity scale (ISS, 0-8, 9-15, 16-25, 226), emergency department (ED) Glasgow coma scale (GCS; 3-8, 9-12, 13-15), normal ED pulse (60-100 beats/minute), normal ED respiratory rate (12-20 breaths/minute), normal ED body temperature $\left(36.6-37.2^{\circ} \mathrm{C}\right)$, normal ED systolic blood pressure (SBP, $90-120 \mathrm{mmHg}$ ), coagulation comorbidity (alcoholism, bleeding disorder, on antithrombotic, or coagulation disorder), bilateral tube placement $(\mathrm{y} / \mathrm{n})$, total hospital length of stay (LOS).

The following information was collected for each tube: date and time of tube placement, date and time of tube removal, was patient discharged with chest tube $(\mathrm{y} / \mathrm{n})$, was video-assisted thoracic surgery (VATS) used for tube placement $(y / n)$, side of tube placement (left anterior, left posterior, right anterior, right posterior), size of chest tube (French scale), initial output of chest tube (milliliters, $\mathrm{mL}$ ), date and time initial output was measured, rate of chest tube output ( $\mathrm{mL} / \mathrm{hour}$ ), and date and time of all chest-tube-related complications (complication must have occurred after tube placement).

\section{Outcome variables}

The primary outcome for this study was having at least one of the following tube complications: 1) requiring a second chest tube (either replacement of initial chest tube or placement of additional tube); 2) tube falling out of patient; 3 ) tube clogging (chest tube clogged by tissue or other material, not allowing blood to drain properly); 4) pneumonia; 5) pleural empyema; 6) and retained HTX (persistent heterogeneous fluid collection detected by computed tomography within 14 days of initial chest tube placement and requiring intervention [second tube or VATS]). Each chest tube complication must have occurred after the placement of the chest tube. The secondary outcomes of interest were 1) individual tube complications; 2) volume of initial chest tube output $(\mathrm{mL})$; 3) rate of initial chest tube output ( $\mathrm{mL} / \mathrm{hr})$; and 4 ) number of hours with each chest tube inserted. The rate of initial chest tube output was calculated by dividing the volume of initial output by the number of hours between chest tube placement and initial output volume measurement.

\section{Statistical analysis}

Because each patient could contribute more than one tube to the study, this study had two levels of analysis: the patient level and the tube level. Patient demographics and injury characteristics were summarized at the patient level, whereas the tube information and tube outcomes were summarized at the tube level. Patientlevel data were analyzed using independent methods, and tube-level data were analyzed using dependent methods, accounting for repeated observations.

Chi-squared tests were used to compare proportions, and Fisher's exact tests were used when more than 50\% of cells had estimated counts less than five. A repeatedmeasures linear regression model was used to compare the mean time each tube was in place and rate of initial output. These models considered the repeated observations, and least squares (LS) means and standard errors (SE) were reported as adjusted measures of central tendency and variability. For non-normal drain rate data, a non-parametric $\mathrm{k}$-sample equality-of-median test was used to test the hypothesis that SB and LB chest tubes were drawn from populations of tubes with similar median drain rates; Fisher's exact $p$-values were calculated. Another linear regression model was used to examine mean differences between chest tube groups in the total time each patient was on all chest tube(s).

One SB tube patient was missing tube size and a value of $12 \mathrm{Fr}$ was imputed. The patient's facility mean and median tube size was 12 Fr. Furthermore, this patient had a total of two chest tubes placed, both by interventional radiology; the first tube was $12 \mathrm{Fr}$ but the second tube was lacking a procedure report for the placement of the second tube and thus the tube size was unknown. The mean, median, and mode for chest tubes placed by interventional radiology at this patient's facility was 11.5 Fr, $12 \mathrm{Fr}$, and $12 \mathrm{Fr}$. Therefore, imputing a tube size of $12 \mathrm{Fr}$ for this chest tube was deemed reasonable with low expectation of misclassification.

The time from tube insertion to output measurement was not precisely recorded for 138 tubes in the electronic medical record $(\mathrm{SB}=91, \mathrm{LB}=47)$, and there were 22 tubes with missing chest tube output volumes. Consequently, there were only 122 tubes for which a 


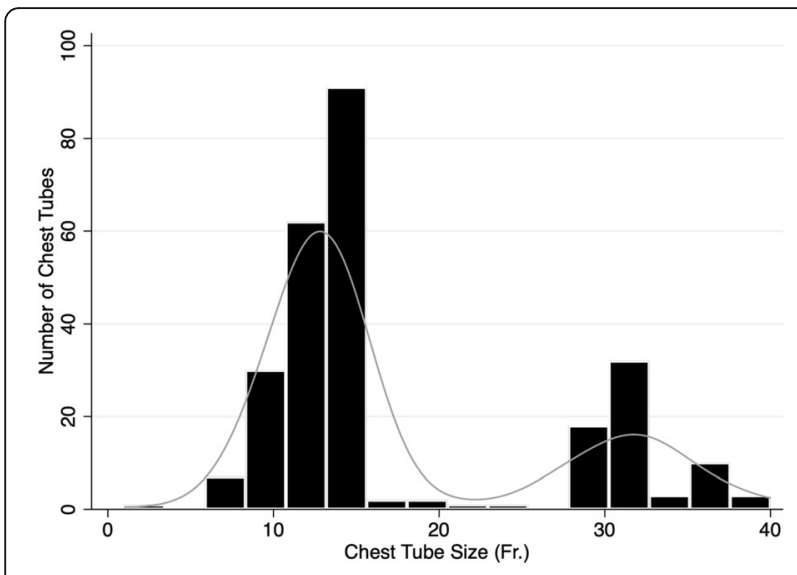

Fig. 1 Distribution of chest tube sizes in study population

drainage rate could be calculated; 100 patients had complete chest tube drainage data, and 120 had missing drainage data for at least one chest tube. The analysis for drainage characteristics is presented only using those 122 chest tubes with drainage rate information. All statistical tests were two-tailed and had an alpha of 0.05; SAS 9.4 (Cary, NC) was used for all analyses.

\section{Results}

There was a total of 220 patients included in our study; 160 patients with SB tubes, and 60 with LB tubes. These patients contributed data on 263 chest tubes; 191 SB tubes, and $72 \mathrm{LB}$ tubes. Figure 1 depicts the histogram for chest tube sizes in the study population. The distribution of LB and SB chest tubes were similar based on placement side of chest (Table 1). There were three patients with bilateral chest tubes. Overall, there were 182 (83\%) patients contributing a single chest tube, 34 (15\%) with two chest tubes, $3(1 \%)$ with three chest tubes, and one patient with four chest tubes $(<1 \%)$; there were no significant differences between tube size groups in the total number of tubes contributed by each patient $(p=$ 0.38 ). The median (IQR) tube size for each group was as follows: SB [12 Fr (12-14) and LB [32 Fr (28-32)].

Overall, there were no significant differences between SB and LB patients in demographics, injury severity, or presenting vital signs (Table 1), except for sex; there was a significantly smaller proportion of males in the SB group, than in the LB group. Sex was not considered a potential confounding variable, nor was it identified in any models as an independent predictor of outcomes. After adjustment for repeated measures, there was no significant difference between LB and SB chest tubes in the length of time each tube was in place [130.13 (41.9) hrs vs. 89.49 (24.1) hrs, $p=0.40$ ]. Table 2 shows where each chest tube was placed and the staff who placed them.

An equivalent proportion of SB and LB chest tubes were placed at the bedside. However, SB chest tubes were more often placed in interventional radiology, whereas LB tubes were more often placed in the operating room (Table 2). Additionally, there were similar proportions of SB and LB chest tubes placed by physicians, surgical residents, and physician assistants. Each patient had an LS mean (SE) total time on chest tubes of 144 (50) hours in the LB group, and 87 (29) hours in the SB group; this difference was not statistically significant (Table 3 ).

Seventeen percent of tubes suffered at least one complication. The results of the primary outcome analysis suggested no significant difference between chest tube groups in the risk of having at least one tube complication (Table 3). The major significant difference in tuberelated complications between chest tube groups were due to VATS and pneumonia events (Table 3). Although LB chest tubes carried a significantly higher risk of subsequent VATS, SB chest tubes had a higher risk of pneumonia. The size of tubes that subsequently required VATS were 32, 32, 32, and 34 Fr. Meanwhile, the median (IQR) size of tubes associated with pneumonia was $14 \mathrm{Fr}(12-14)$. These study data did not indicate there were significant differences between SB and LB chest tubes in the risk of tubes requiring replacement, falling out, or clogging; or resulting in pleural empyema or retained HTX.

There were notable differences in drainage characteristics between the two tube groups (Table 3). Volume of initial output for SB chest tubes was assessed an average of $7.8 \mathrm{~h}$ later from tube insertion, compared to LB chest tubes $(p<0.001)$. There was no significant difference in the mean initial output volume between LB and SB chest tubes. Ultimately, LB chest tubes had a drainage rate that was 4 -fold faster than $\mathrm{SB}$ chest tubes $(213.4 \mathrm{~mL} / \mathrm{hr}$. vs. $52.2 \mathrm{~mL} / \mathrm{hr}$., $\mathrm{p}<0.001)$. According to a non-parametric test of medians, there was no difference between LB and SB chest tubes in the median (IQR) drainage rates (39.7 [23.5-242.0] $\mathrm{mL} / \mathrm{hr}$. vs. 38.6 [27.5-53.8], $p=0.81$ ). Demographic and clinical characteristics were compared between patients with complete chest tube drainage information and those missing drainage data (Supplemental Table S1). Patients with complete data had significantly lower proportions of patients aged 18-29years, and higher proportions of patients aged 70-89 years. Additionally, patients with complete drainage data were less severely injured according to the injury severity scale. Lastly, there was a higher proportion of patients with complete data who had normal body temperature at admission, compared to patients with missing drainage data.

Nevertheless, both tube size groups had similar mean total lengths of time with chest tubes throughout their hospitalization. Finally, there were no significant differences between chest tube groups in LS mean hospital LOS nor in the proportion of patients who returned to prior lung function. 
Table 1 Demographic and clinical characteristics of 220 patients with delayed hemothorax

\begin{tabular}{|c|c|c|c|}
\hline Variable, n(\%) & Large-Bore $(n=60)$ & Small-Bore $(n=160)$ & $P$ \\
\hline Male & $51(85 \%)$ & $112(70 \%)$ & 0.02 \\
\hline Age, years & & & 0.67 \\
\hline 18 to 29 & $6(10 \%)$ & $13(8 \%)$ & \\
\hline 30 to 49 & $10(17 \%)$ & $38(24 \%)$ & \\
\hline 50 to 69 & $25(42 \%)$ & $69(43 \%)$ & \\
\hline 70 to 89 & $18(30 \%)$ & $36(23 \%)$ & \\
\hline $90+$ & $1(2 \%)$ & $4(3 \%)$ & \\
\hline Injury type & & & 0.11 \\
\hline Blunt & $54(95 \%)$ & 158 (99\%) & \\
\hline Penetrating & $3(5 \%)$ & $2(1 \%)$ & \\
\hline Highest chest AIS value & & & 0.28 \\
\hline$<3$ & $4(9 \%)$ & $20(15 \%)$ & \\
\hline$\geq 3$ & $43(91 \%)$ & $117(85 \%)$ & \\
\hline Injury severity scale & & & 0.57 \\
\hline 0 to 8 & 0 & $3(2 \%)$ & \\
\hline 9 to 15 & $21(35 \%)$ & $68(43 \%)$ & \\
\hline 16 to 25 & $24(24 \%)$ & $53(33 \%)$ & \\
\hline $26+$ & $15(25 \%)$ & $35(22 \%)$ & \\
\hline ED Glasgow coma scale & & & 0.22 \\
\hline 3 to 8 & $8(16 \%)$ & $14(9 \%)$ & \\
\hline 9 to 12 & $2(4 \%)$ & $3(2 \%)$ & \\
\hline 13 to 15 & $41(80 \%)$ & $140(89 \%)$ & \\
\hline \multicolumn{4}{|l|}{ ED vital signs ${ }^{a}$} \\
\hline Normal pulse & 27 (71\%) & $11(70 \%)$ & 0.86 \\
\hline Normal respiratory rate & $38(75 \%)$ & $121(79 \%)$ & 0.55 \\
\hline Normal body temperature & $23(48 \%)$ & $62(47 \%)$ & 0.94 \\
\hline Normal systolic blood pressure & $8(20 \%)$ & $473(30 \%)$ & 0.21 \\
\hline Coagulation comorbidity ${ }^{b}$ & $10(17 \%)$ & $31(19 \%)$ & 0.65 \\
\hline Bilateral chest tubes & $1(2 \%)$ & $2(1 \%)$ & 0.81 \\
\hline Tube placement, side of chest ${ }^{c}$ & & & 0.68 \\
\hline Right & $34(49 \%)$ & $98(52 \%)$ & \\
\hline Left & $36(51 \%)$ & $91(48 \%)$ & \\
\hline Median (IQR) time from admission until first chest tube, hours ${ }^{c}$ & $111(80-192)$ & $109(80-176)$ & 0.56 \\
\hline
\end{tabular}

AIS, abbreviated injury scale; ED, emergency department; IQR, interquartile range. Column totals may not add to group total due to missing data

${ }^{a}$ Normal pulse (60-100 beats per minute); normal respiratory rate (12-20 breaths per minute); normal body temperature (36.6-37.2 C); normal blood pressure (SBP 90-120 mmHg)

${ }^{\mathrm{b}}$ Coagulation comorbidity defined as alcoholism, bleeding disorder, on clopidogrel, coagulation disorder

c Calculated based on each tube

\section{Discussion}

In the setting of delayed HTXs, this is one of the first studies to include an examination of SB chest tubes smaller than $14 \operatorname{Fr}[6-8,11,15]$ and contributes the history of incremental reporting of increasingly smaller chest tube diameters. According to the data, there were no significant differences between chest tubes $\leq 14 \mathrm{Fr}$ vs. $>14 \mathrm{Fr}$ in the probability of experiencing at least one chest tube complication (Table 3). Furthermore, there were no major differences between groups in the drainage rate or volume, though drainage rate data should be interpreted with caution.

The current study used a different primary outcome compared to previous studies, with the aim of providing a more global perspective on chest tube outcomes, $[6,8$, 13 ] and most closely resembles the primary outcome presented in the Tanizaki et al. study [11]. Previously, tube-related outcomes have been separated into failures (e.g. VATS, requiring a second tube) and complications 
Table 2 Chest tube groups and complications, by insertion location and staff

\begin{tabular}{|c|c|c|c|c|c|}
\hline N (\%) & $\begin{array}{l}\text { Large-Bore } \\
(n=72)\end{array}$ & $\begin{array}{l}\text { Small-Bore } \\
(n=191)\end{array}$ & $P$ & $\begin{array}{l}\text { Any Tube Complication } \\
(n=45)\end{array}$ & $P$ \\
\hline Location of tube placement & & & $<0.001$ & & 0.91 \\
\hline Bedside & $32(44 \%)$ & $86(45 \%)$ & & $19(16 \%)$ & \\
\hline Interventional radiology & $6(8 \%)$ & $100(52 \%)$ & & 19 (18\%) & \\
\hline Operating room & $34(47 \%)$ & $4(2 \%)$ & & $7(8 \%)$ & \\
\hline Staff who placed tube & & & 0.10 & & 0.20 \\
\hline Physician & $51(71 \%)$ & 137 (72\%) & & $36(19 \%)$ & \\
\hline Surgical resident & $2(3 \%)$ & $18(9 \%)$ & & $5(9 \%)$ & \\
\hline Physician assistant & $19(26 \%)$ & $36(19 \%)$ & & $4(20 \%)$ & \\
\hline
\end{tabular}

(e.g. tube fell out, clogging of tube, retained HTX, pneumonia, pleural empyema). Instead, the current study considered each of these outcomes a tube complication. We believe this allowed for an overall comparison of risk of complication between SB and LB chest tubes. Despite the difference in definition of the primary endpoint in the current study, these results were consistent with multiple studies examining the use of SB chest tubes for the management of HTXs, particularly with respect to the higher risk of VATS with the use of LB chest tubes $[6,8,11]$.

On the contrary, unlike previously published studies on the topic, the current study documented an increased risk of pneumonia with the use of SB chest tubes in the setting of delayed HTXs $[6-8,11,19]$. It is possible that the development of pneumonia could have been precipitated by the development of another or previous complication, including a retained HTX or pleural empyema. However, of the 14 patients who developed pneumonia, only two suffered another tube complication: one patient had a tube replaced, and the second patient's chest tube fell out. Therefore, there was no clear link between the development of pneumonia and a previous or concomitant complication. A possible cause of the increased risk of pneumonia could be related to the number of rib fractures or ventilator days $[20,21]$; however, neither were available for data analysis. Furthermore, due to limitations in drainage rate data and the fact that all pneumonia events occurred in the SB chest tube group, we could not investigate the relationship between chest tube

Table 3 Tube and patient outcomes by comparison groups

\begin{tabular}{|c|c|c|c|}
\hline Variable, n (\%) & Large-Bore & Small-Bore & $P$ \\
\hline Tube outcomes & $\mathrm{n}=72$ & $n=191$ & \\
\hline$\geq 1$ tube complication & $10(14 \%)$ & $35(18 \%)$ & 0.42 \\
\hline Tube replaced & $6(8 \%)$ & $8(4 \%)$ & 0.22 \\
\hline VATS & $4(6 \%)$ & 0 & 0.005 \\
\hline Tube fell out & 0 & $7(4 \%)$ & 0.20 \\
\hline Tube clogged & $1(1 \%)$ & $8(4 \%)$ & 0.45 \\
\hline Pneumonia & 0 & $14(7 \%)$ & 0.01 \\
\hline Pleural empyema & $1(1 \%)$ & $2(1 \%)$ & $>0.99$ \\
\hline Retained hemothorax & $3(4 \%)$ & $5(3 \%)$ & 0.69 \\
\hline Drainage metrics & $n=22$ & $n=100$ & \\
\hline Time from chest tube insertion until initial output measurement, LS mean (SE), hr & $12.8(1.75)$ & $20.6(0.86)$ & $<0.001$ \\
\hline Initial output, LS mean (SE), mL & $738.0(121.56)$ & $810.9(57.02)$ & 0.59 \\
\hline Rate of initial output, LS mean (SE), mL/hr & $213.4(32.98)$ & $52.2(15.47)$ & $<0.001$ \\
\hline Patient outcomes & $\mathrm{n}=60$ & $n=160$ & \\
\hline Total time on chest tube(s), LS mean (SE), hr & $144.6(50.05)$ & $86.5(28.80)$ & 0.32 \\
\hline Hospital LOS, LS mean (SE), day & $15.0(1.26)$ & $15.1(0.73)$ & 0.95 \\
\hline Patient returned to prior lung function ${ }^{\text {a }}$ & $25(42 \%)$ & $42(36 \%)$ & 0.43 \\
\hline
\end{tabular}

VATS, video assisted thoracic surgery; $\mathrm{Cl}$, confidence interval; LS, least squares; SE, standard error; IQR, interquartile range; $\mathrm{mL}$, milliliters; LOS, length of stay

${ }^{a}$ Assessed at discharge by physical therapy; 42 small-bore patients missing data 
drainage rate and the development of pneumonia. It is also possible this finding is due to chance alone. This potentially serious risk must be weighed against the observed benefits of SB chest tubes.

Anecdotally, LB chest tubes are preferred to SB tubes primarily because they are thought to more readily drain a HTX. The current study examined the initial output volume and rate of initial output for each chest tube. According to the current study, LB chest tubes were not significantly different from $\mathrm{SB}$ chest tubes regarding the volume of initial output (Table 3); this finding is consistent across the literature on thoracic trauma $[6-8,10,11$, 14]. According to theory, a $28 \mathrm{Fr}$ chest tube should have roughly 13 -fold the draining capacity of a 14 Fr chest tube [18]; the current study data contradict this notion. Caution is necessary when interpreting the tube drainage rate information, as it comes from a subset $(n=122)$ of the larger study population. Additionally, a lack of consistency in measuring time from chest tube insertion to output volume measurement likely caused inaccurate estimations of the drainage rates, likely secondary to inaccurate times in the denominators of each rates. This is plausible given the lack of control in data collection with a retrospective study. Nevertheless, a slower drainage rate by SB chest tubes might not preclude their use in the setting of delayed HTX. Slow pleural drainage rates are likely to be problematic in the setting of acute traumatic HTXs which require rapid evacuation of pleural blood. But, in the setting of delayed or non-emergent HTXs, the slower drainage of SB chest tubes might be an acceptable tradeoff given their potential benefits vis-à-vis lowered pain $[15,17]$.

The current study had lower power to detect differences between chest tube groups in individual complications. However, an absence of evidence is not evidence of absence, making it important to consider nonsignificant results. Despite non-significance, SB tubes had a nominal 4-fold higher risk of falling out and clogging when compared to their LB counterparts. This is supported by other studies documenting increased risks of SB chest tubes clogging and falling out [12, 17]. It therefore remains important to consider these risks and implement mitigating strategies to prevent their occurrence when using SB chest tubes. These include the development of novel techniques for securing chest tubes to the chest wall, and the frequent flushing of SB chest tubes with saline [12].

It is necessary to consider the limitations of this study. First, the retrospective non-randomized nature of this study limits its generalizability and proximity to elucidating causal associations. One facility in this multi-center collaboration routinely placed SB chest tubes for delayed HTXs, while the other five centers routinely placed LB chest tubes. Owing to the lack of differences between chest tube size groups in demographic, clinical, and injury characteristics, we believe these results are not heavily influenced by selection bias between the study hospitals; nevertheless, it cannot be completely dismissed. Because the success of a chest tube is dictated by correct placement, the current study is favorable because it included the practice variability for LB chest tube placement across five Level I Trauma Centers; however, there is less practice variability in the placement of the SB chest tubes as they were placed primarily at a single hospital. Despite the non-randomized nature of this study, both study groups were similar in terms of demographics, overall injury characteristics, the mean time from admission until first chest tube placement, and the mean duration with a chest tube. The lack of treatment randomization allows for residual confounding and unmeasured confounding to be possible contributors to the results in the current study. Furthermore, due to the limitations of patient chart review, the amount of time that lapsed between chest tube placement and chest tube output measurement was not precisely recorded for a majority of patients in this study, and results from the analysis of mean drainage rates should not be over interpreted; general conclusions should be drawn from the non-parametric comparison of median drainage rates. Owing to the limitations inherent with retrospective cohort studies, these results should be confirmed in a randomized clinical trial. Accurately assessing drainage rates is vital to comparing the performance of SB and LB chest tubes and should be consistently measured in future prospective observational and randomized study designs.

\section{Conclusions}

The current study described the complication and drainage characteristics of SB and LB chest tubes in the management of delayed HTXs. Compared to LB chest tubes, there was no clear overall benefit or harm to the use of SB in the setting of delayed HTXs. Because of the continued equipoise regarding the use of SB versus LB chest tubes, there remains a need for a randomized clinical trial. If SB chest tubes are used to treat delayed HTXs, physicians must be vigilant to the development of pneumonia and the potential for the chest tubes to become dislodged or clogged.

\section{Supplementary information}

Supplementary information accompanies this paper at https://doi.org/10. 1186/s13049-020-00754-5.

Additional file 1: Table S1. Comparison of demographic and clinical characteristics between patients with and without missing chest tube drainage data

\section{Abbreviations}

HTX: Hemothorax; LB: Large-bore; SB: Small-bore; Fr: French;

ICD: International classification of disease; AIS: Abbreviated injury scale; 
ISS: Injury severity scale; ED: Emergency department; GCS: Glasgow coma scale; C: Celsius; SBP: Systolic blood pressure; VATS: Video-assisted thoracic surgery; mL: Milliliters; LS: Least squares; SE: Standard error; NC: North Carolina (USA); HIPAA: Heath Insurance Portability and Accountability Act; IQR: Interquartile range; LOS: Length of stay

\section{Acknowledgements}

We would like to thank the following clinical research coordinators for their hard work and diligent data collection for this study: Jennifer Pekarek, Diane Redmond, Kathy Rodkey, Carolyn Blue, Jamie Shaddix, and Shenequa Deas. We would also like to thank Tina Thompson for her excellent work in managing our submissions to the IRBs of all six collaborating centers.

\section{Authors' information (optional)}

Not applicable.

\section{Authors' contributions}

AO contributed to the design of the study; acquisition, analysis, and interpretation of data; drafting and revising of the manuscript; final approval of the manuscript version to be published; and agreeing to be accountable for all aspects of work. JC contributed to the conception and study design; drafting and revising of the manuscript; final approval of the manuscript version to be published; and agreeing to be accountable for all aspects of work. MC contributed to the interpretation of data; revising of the manuscript; final approval of the manuscript version to be published; and agreeing to be accountable for all aspects of work. AHT contributed to the interpretation of data; revising of the manuscript; final approval of the manuscript version to be published; and agreeing to be accountable for all aspects of work. KB contributed to the interpretation of data; revising of the manuscript; final approval of the manuscript version to be published; and agreeing to be accountable for all aspects of work. RV contributed to the interpretation of data; revising of the manuscript; final approval of the manuscript version to be published; and agreeing to be accountable for all aspects of work. LM contributed to the interpretation of data; revising of the manuscript; final approval of the manuscript version to be published; and agreeing to be accountable for all aspects of work. AD contributed to the interpretation of data; revising of the manuscript; final approval of the manuscript version to be published; and agreeing to be accountable for all aspects of work. DBO contributed to the design of the study; interpretation of data; revising of the manuscript; final approval of the manuscript version to be published; and agreeing to be accountable for all aspects of work.

\section{Funding}

We would like to thank and recognize the following hospitals for their financial and administrative support for this study: Swedish Medical Center (Englewood, Colorado, USA); St. Anthony Hospital (Lakewood, Colorado, USA); Medical City Plano (Plano, Texas, USA); Penrose-St. Francis Hospital (Colorado Springs, Colorado, USA); Research Medical Center (Kansas City, Missouri, USA); and Wesley Medical Center (Wichita, Kansas, USA).

\section{Availability of data and materials}

The datasets used and/or analyzed during the current study are available from the corresponding author on reasonable request.

\section{Ethics approval and consent to participate}

This study was approved by each facility's institutional review board and was granted a waiver of informed consent and HIPAA authorization.

\section{Consent for publication}

Not applicable.

\section{Competing interests}

All authors state that they have no conflicts of interest to declare.

\section{Author details}

${ }^{1}$ St. Anthony Hospital, Lakewood, CO, USA. ${ }^{2}$ Swedish Medical Center, Englewood, CO, USA. ${ }^{3}$ Medical City Plano, Plano, TX, USA. ${ }^{4}$ Penrose Hospital, Colorado Springs, Colorado Springs, CO, USA. ${ }^{5}$ Research Medical Center, Kansas City, MO, USA. ${ }^{6}$ Wesley Medical Center, Wichita, KS, USA.
Received: 23 March 2020 Accepted: 12 June 2020

Published online: 22 June 2020

\section{References}

1. Khandhar SJ, Johnson SB. Calhoon JH. Thoracic Surgery Clinics: Overview of Thoracic Trauma in the United States; 2007.

2. Richardson JD, Miller FB, Carrillo EH, Spain DA. COMPLEX THORACIC INJURIES. Surg Clin North Am 1996 Aug;76(4):725-748.

3. Sharma OP, Hagler S, Oswanski MF. Prevalence of delayed hemothorax in blunt thoracic trauma. Am Surg. 2005;71(6):481-6.

4. Misthos P, Kakaris S, Sepsas E, Athanassiadi K, Skottis I. A prospective analysis of occult pneumothorax, delayed pneumothorax and delayed hemothorax after minor blunt thoracic trauma $\hat{z}^{2}$. Eur J Cardio-Thoracic Surg. 2004 May; 25(5):859-64.

5. Mattox KL, Wall MJ, Tsai P. Chapter 24. Trauma thoracotomy: principles and techniques. In: Mattox KL, Moore EE, Feliciano D V, editors. Trauma. 7th ed. New York, NY: The McGraw-Hill Companies; 2013.

6. Bauman ZM, Kulvatunyou N, Joseph B, Jain A, Friese RS, Gries L, et al. A prospective study of 7-year experience using percutaneous 14-French pigtail catheters for traumatic Hemothorax/Hemopneumothorax at a Level1 trauma center: size still does not matter. World J Surg. 2018 Jan 9;42(1): 107-13.

7. Inaba K, Lustenberger T, Recinos G, Georgiou C, Velmahos GC, Brown C, et al. Does size matter? A prospective analysis of 28-32 versus 36-40 French chest tube size in trauma. J Trauma Acute Care Surg. 2012 Feb;72(2):422-7.

8. Kulvatunyou N, Joseph B, Friese RS, Green D, Gries L, O'Keeffe T, et al. 14 French pigtail catheters placed by surgeons to drain blood on trauma patients: is 14-Fr too small? J Trauma Acute Care Surg. 2012;73(6):1423-7.

9. Mendes MA, China Pereira N, Ribeiro C, Vanzeller M, Shiang T, Gaio R, et al. Conventional versus pigtail chest tube-are they similar for treatment of malignant pleural effusions? Support Care Cancer. 2018 Aug 30;26(8):2499-502.

10. Russo RM, Zakaluzny SA, Neff LP, Grayson JK, Hight RA, Galante JM, et al. A pilot study of chest tube versus pigtail catheter drainage of acute hemothorax in swine. J Trauma Acute Care Surg. 2015 Jun;79(6):1038-43.

11. Tanizaki S, Maeda S, Sera M, Nagai H, Hayashi M, Azuma H, et al. Small tube thoracostomy (20-22 Fr) in emergent management of chest trauma. Injury. 2017 Sep;48(9):1884-7.

12. Mahmood K, Wahidi MM. Straightening out chest tubes: what size, what type, and when. Clin Chest Med. 2013;34(1):63-71.

13. Lepsen UW, Ringbæk T. Small-bore chest tubes seem to perform better than larger tubes in treatment of spontaneous pneumothorax. Dan Med J. 2013.

14. Korczyński P, Górska K, Nasiłowski J, Chazan R, Krenke R. Comparison of small bore catheter aspiration and chest tube drainage in the Management of Spontaneous Pneumothorax. Adv Exp Med Biol. 2015:15-23.

15. Kulvatunyou N, Erickson L, Vijayasekaran A, Gries L, Joseph B, Friese RF, et al. Randomized clinical trial of pigtail catheter versus chest tube in injured patients with uncomplicated traumatic pneumothorax. Br J Surg. 2014 Jan; 101(2):17-22.

16. Rahman NM, Maskell NA, Davies CWH, Hedley EL, Nunn AJ, Gleeson FV, et al. The relationship between chest tube size and clinical outcome in pleural infection. Chest. 2010 Mar;137(3):536-43.

17. Rahman NM, Pepperell J, Rehal S, Saba T, Tang A, Ali N, et al. Effect of opioids vs NSAIDs and larger vs smaller chest tube size on pain control and pleurodesis efficacy among patients with malignant pleural effusion: the TIME1 randomized clinical trial. JAMA - J Am Med Assoc. 2015;314(24):2641-53.

18. Niinami $H$, Tabata M, Takeuchi $Y$, Umezu M. Experimental assessment of the drainage capacity of small Silastic chest drains. Asian Cardiovasc Thorac Ann. 2006 Jun 26;14(3):223-6.

19. Rivera L, O'Reilly EB, Sise MJ, Norton VC, Sise CB, Sack DI, et al. Small catheter tube Thoracostomy: effective in managing chest trauma in stable patients. J Trauma Inj Infect Crit Care. 2009 Feb;66(2):393-9.

20. Bulger EM, Arneson MA, Mock CN, Jurkovich GJ. Rib fractures in the elderly. J Trauma Inj Infect Crit Care. 2000 Jun;48(6):1040-7.

21. Benns MV, Egger ME, Harbrecht BG, Franklin GA, Smith JW, Miller KR, et al. Does chest tube location matter? An analysis of chest tube position and the need for secondary interventions. J Trauma Acute Care Surg. 2015;78(2): 386-90.

\section{Publisher's Note}

Springer Nature remains neutral with regard to jurisdictional claims in published maps and institutional affiliations. 